\section{Der $\mathrm{p} 1$ dreidimensional}

Für die exakte Aufklärung der molekularen Mechanismen bei der IgE-vermittelten Aktivierung von Effektorzellen ist die Kenntnis der dreidimensionalen Proteinstruktur von Allergenen unerlässlich. Nun wurde erstmals eines der Hauptallergene der Hausstaubmilbe, Der p 1, per Röntgenstrukturanalyse untersucht.

ei fast allen Patienten mit einer Hausstaubmilbenallergie kann spezifisches IgE gegen Der p 1, das Hauptallergen von Dermatophagoides pteronyssinus, nachgewiesen werden. Der $\mathrm{p} 1$ ist eine Zysteinprotease, die im Verdauungstrakt der Milbe vorkommt und sich im Milbenkot in hohen Konzentrationen anreichert. Die proteolytische Aktivität dieses Proteins trägt höchstwahrscheinlich zu dem hohen Sensibilisierungspotenzial von Der p 1 bei.

Bei der Aufklärung der dreidimensionalen Struktur von Proteinen wird häufig auf die Röntgenkristallographie der im festen Zustand vorliegenden Proteine zurückgegriffen. Um ein möglichst reines Molekül für die Kristallbildung zu

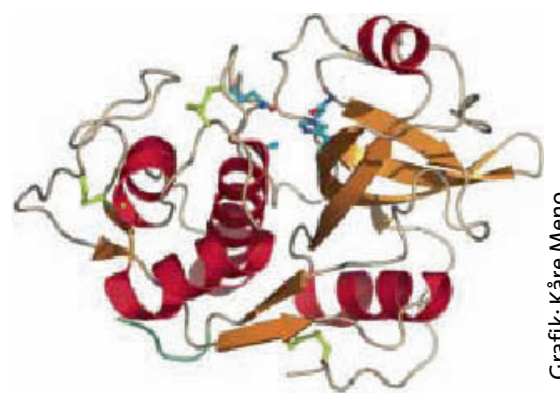

Gesamtstruktur von rproDer $p$ 1, einer rekombinanten Variante des Milbenallergens Der $p$ 1. $\alpha$-Helices sind als rote Spiralen, $\beta$-Stränge als orange Pfeile und Disulfidbrücken als gelbe Stäbe dargestellt.

erhalten, wurde in der vorliegenden Untersuchung ein modifiziertes rekombi- nantes Molekül, rproDer p 1, eingesetzt. Daten im Auflösungsbereich von 1,61$11 \AA \AA$ wurden verwendet.

In weiteren Untersuchungen mit Immunelektrophorese und IgE-Inhibitionstests zeigte sich, dass einige der gefundenen Epitope des rekombinanten Moleküls dem natürlich vorkommenden Der p 1 entsprechen. Die Analyse verschiedener Propeptide des Moleküls ergab außerdem neue Strukturen, die die Zuordnung von Der p 1 innerhalb der Cysteinprotease-Familie 1 in eine neue dritte C1-Subfamilie nahelegen.

Fazit: Mit der Aufklärung der dreidimensionalen Struktur von Der p 1 können die immunologischen Sensibilisierungsmechanismen besser verstanden werden. Außerdem erlauben die Kenntnisse möglicherweise die Herstellung von Strukturvarianten für die spezifische Immuntherapie.

$b k$

Meno K et al. The crystal structure of recombinant proDer $\mathrm{p} 1$, a major house dust mite proteolytic allergen. J Immunol 2005; 175: 3835-45

\title{
Wer lässt die Allergene aus den Pollen?
}

\section{Pollenallergiekranke sind gegen Proteine allergisch, die bei Kontakt eines Pollenkorns mit Feuchtigkeit freigesetzt werden. Das Ver- ständnis dieses Prozesses ist leider noch recht unvollständig.}

$\mathrm{B}$ iologen der österreichischen Universität Salzburg wählten für ihr Experiment zur Kinetik der Proteinfreisetzung die hochallergenen Pollenkörner von Artemisia vulgaris und die kaum allergisierenden Pollenkörner von Lilium longiflorum. Die Proteinfreisetzung bei Kontakt mit drei verschiedenen Flüssigkeiten wurde per Chromatographie verfolgt: Eine phosphatgepufferte physiologische Kochsalzlösung sollte die $\mathrm{pH}$ - und Ionenverhältnisse der menschlichen $\mathrm{Na}$ senschleimhaut imitieren, destilliertes Wasser einen Regenguss und ein gebräuchliches Keimmedium die Verhältnisse bei der natürlichen Pflanzenbefruchtung.

Bei beiden Pollenspezies zeigte sich eine zu Beginn des Flüssigkeitskontakts rasch einsetzende und dann exponentiell abnehmende Freisetzung von Gesamtprotein und der zellwandständigen sauren Phosphatase. Dies spricht für einen diffusionsgesteuerten Mechanismus, bei dem überwiegend Substanzen der äußeren Zellwandkompartimente ausgeschwemmt werden. Die Elutionsprofile von Profilin und Art v 1 als Stellvertreter für sensibilisierend wirkende Moleküle wiesen dagegen keine Diffusionscharakteristik auf. Das gleiche galt für das nicht allergisierende 14-3-3-Protein. Die Freisetzung dieser Proteine war unabhängig von $\mathrm{pH}$-Wert, Osmolarität oder Salzkonzentration der Elutionsflüssigkeit.

Die Freisetzungskinetik der Allergene deutet damit auf einen von äuße- ren Bedingungen unabhängigen Mechanismus, der entsprechend der physiologischen Funktion der Proteine bei der Befruchtung in Gang gesetzt wird. Die Autoren vermuten, dass es sich hierbei um einen aktiven sekretorischen Prozess handelt. Dieser findet aber nur in wässrigem Milieu statt, 100\%iges Glycerin verhindert die Allergenfreisetzung.

Fazit: Bei der Allergenfreisetzung aus Pollenkörnern spielen neben einer Diffusion auch aktive Transportprozesse vom Zytoplasma in die Umgebung eine Rolle. Wenn diese Prozesse entschlüsselt wären, könnte versucht werden, durch eine entsprechende therapeutische Intervention die Allergenfreisetzung zu verhindern.

$b k$

Hoidn C et al. Nondiffusional release of allergens from pollen grains of Artemisia vulgaris and Lilium longiflorum depends mainly on the type of the allergen. Int Arch Allergy Immunol 2005; 137: 27-36 